\title{
Radar Cross Sections of Plasma Bodies at the Plasma Frequency ${ }^{1}$
}

\author{
Leon Peters, Jr. \\ Contribution From the Antenna Laboratory, Department of Electrical Engineering, The Ohio State \\ University, Columbus, Ohio
}

(Received September 3, 1964; revised October 2, 1964

\begin{abstract}
Two components of the radar cross section of a plasma body at the plasma frequency are considered. These are the specular reflection and the creeping wave components. The specular component is obtained by use of the Fresnel reflection coefficient and the creeping wave component from that for the perfectly conducting body which is adjusted by means of a loss attenuation factor.

The method is applied to the sphere and the resulting cross sections compare favorably with those obtained using the exact solution. These methods can be applied to smooth bodies other than those of spherical shape and to dielectric-constants other than zero provided the creeping wave component for the perfectly conducting body is known.
\end{abstract}

\section{Introduction}

The radar cross section of plasma bodies at a frequency equal to the plasma frequency is controlled by means of the loss mechanism. The manner in which it is controlled is to be demonstrated in this paper by consideration of lossy homogeneous plasma spheres.

The exact boundary solution for the scattering of electromagnetic waves from a spherical body has been treated [Mie, 1908]. The exact solution is to be used to verify the approximate solutions for this plasma body at the plasma frequency. Many approximate solutions for conducting bodies have appeared recently which involve a specular reflection and a wave known as the creeping wave [Franz and Depperman, 1954 a and b; Keller and Levy, 1959] which travels around the body and then radiates in the direction of the tangent as illustrated in figure 1 for the case of backscatter.

\footnotetext{
1 The work reported in this paper was supported in part by Contract Number AF 33(616)-8039 between Research Technology Division, Air Force Avionics Laboratory, Wright-Patterson Air Force Base, Ohio and The Ohio State University Research Foundation.
}

It is this treatment that leads to an understanding of the scattering mechanisms at the plasma frequency. The attenuation factor for the Zenneck wave traveling along a lossy planar interface shown in figure 2 is to be used to estimate the loss introduced into the creeping wave component. This yields approximately the magnitude for this component, once its magnitude is known for the conducting body. The characteristics of the creeping wave and the Zenneck wave in general differ. However the magnitude of the attenuation factor is proportional to the percentage of energy propagating in the lossy medium. These two types of waves have field distributions near the boundary that are similar and thus it is postulated that the introduction of finite conductivity would cause approximately the same percentage of energy to flow within the lossy medium and thus yield approximately the same attenuation factor due to the loss mechanism. The validity of this approximation is demonstrated by the Mie solution as noted previously. An alternative more rigorous approach would consist of a solution for lossy bodies paralleling the creeping wave solution for the perfectly conducting bodies. Such an approach certainly merits consideration, and in this connection, Wait [1964] gives an excellent summary of the cases that have been treated in this manner in his treatment of a radial dipole over a sphere.

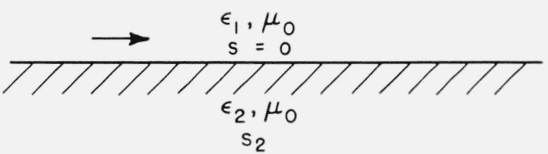

FIGURE 2. Wave propagating along lossy interface. 

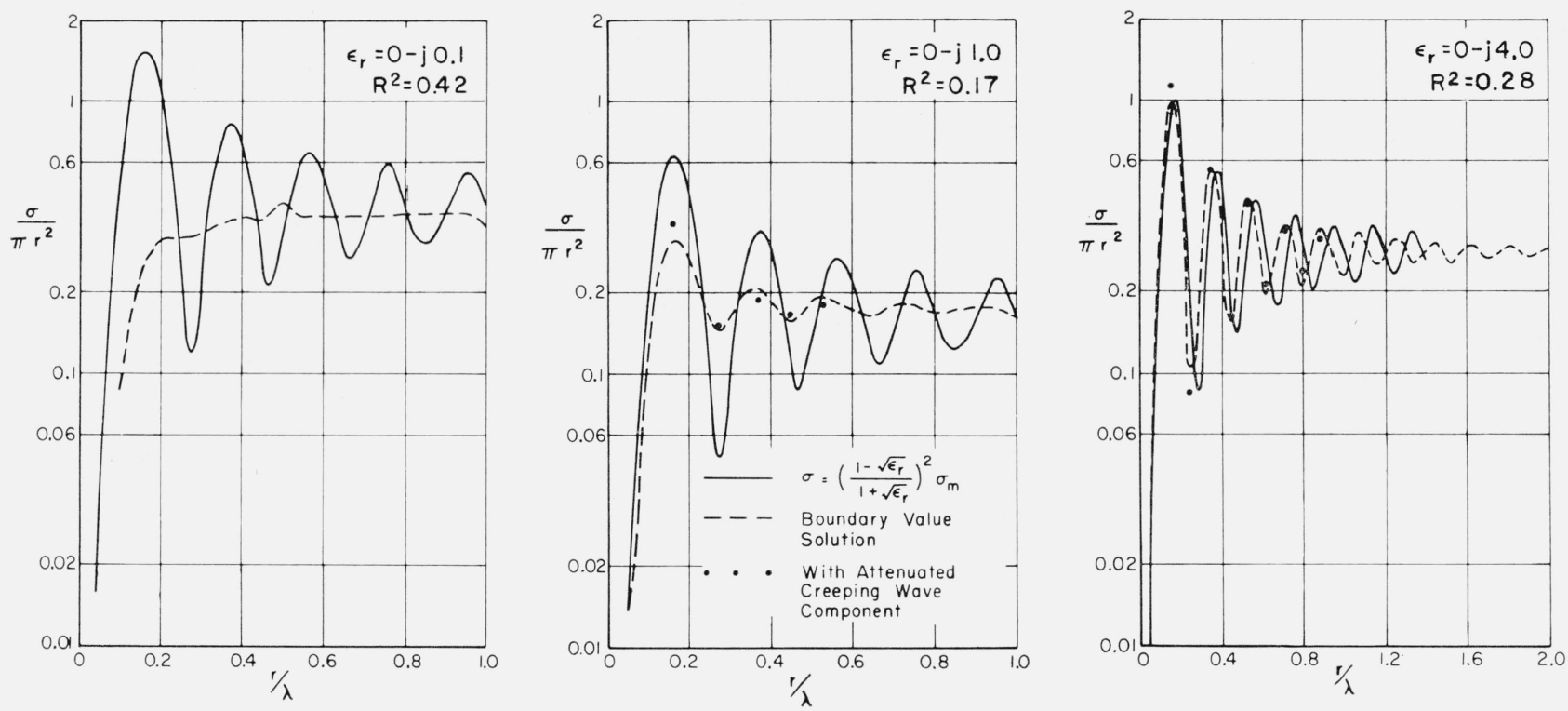

Figure 3. Radar cross sections of lossy plasma spheres.

Also the creeping wave solution would be applicable only to such classical shapes as the sphere and cylinder. The present approach is comparatively independent of geometry since the method was evolved for a planar interface and applied without modification to the sphere once the creeping wave for the perfectly conducting case is known. (See fig. 2.)

A simpler alternate approximate solution would take the form of the radar cross section of the perfectly conducting body times the square of the reflection coefficient but this solution is not always effective.

\section{Approximate Solutions}

The simplest solution for the radar cross section is

where

$$
\sigma=R^{2} \sigma_{\infty},
$$

$\sigma=$ radar cross section,

$\sigma_{\infty}=$ radar cross section of the perfectly conducting body of the same size and shape.

$R=\frac{1-n}{1+n}=$ Fresnel reflection coefficient, and

$n=$ the complex index of refraction.

The complex index of refraction $n$ is defined by

$$
\begin{aligned}
\gamma=j \beta_{0} n & =j \beta_{0} \sqrt{\epsilon_{r}}=\sqrt{j \omega \mu s-\omega^{2} \mu \epsilon^{\prime}} \\
& =j \omega \sqrt{\mu \epsilon_{0}} \sqrt{\epsilon_{r}^{\prime}-j \frac{s}{\omega \epsilon_{0}}}
\end{aligned}
$$

where

$\gamma=$ propagation constant,

$\omega=$ angular frequency,

$\epsilon^{\prime}=$ dielectric constant,

$\epsilon_{r}^{\prime}=\epsilon^{\prime} / \epsilon_{0}$

$\epsilon_{0}=$ dielectric constant of free space,

$s=$ conductivity,

$\mu=$ permeability,

$\beta_{0}=\omega \sqrt{\mu \epsilon_{0}}=$ phase factor of free space, and

$\boldsymbol{\epsilon}_{r}=\boldsymbol{\epsilon}_{r}^{\prime}-j \boldsymbol{\epsilon}_{r}^{\prime \prime}=$ the complex dielectric constant.

The complex dielectric constant

$$
\begin{aligned}
\boldsymbol{\epsilon}_{r}=n^{2} & =\epsilon_{r}^{\prime}-j \frac{s}{\omega \epsilon_{0}} \\
& =\epsilon_{r}^{\prime}(1-j \tan \delta)
\end{aligned}
$$

where

$$
\tan \delta=\text { loss tangent }=\frac{s}{\omega \epsilon^{\prime}} .
$$

The approximation of (2) assumes that all of the energy transmitted into the body is absorbed. The remainder of the energy is then reflected by the body acting as though it were a perfect conductor.

The results of this approximate solution are compared in figure 3 with those obtained from the boundary value solution for three cases; $\epsilon_{r}=0-$ $j 0.1,0-j 1.0$, and $0-j 4.0$. These three cases are for a wave incident on a lossy plasma sphere with a frequency equal to the plasma cutoff frequency.

This first order approximation may be improved by considering the reflection mechanisms individually. The specular component of scattered fields is 
readily calculated by multiplying the scattered field obtained from geometrical optics by the square of the reflection coefficient or

where

$$
\sigma_{\mathrm{sp}}=R^{2} \sigma_{\mathrm{opt}}
$$

$\sigma_{\mathrm{sp}}=$ specular component of radar cross section, and $\sigma_{\mathrm{opt}}=$ geometrical optics radar cross section of the perfectly conducting body.

The geometrical radar cross section for a sphere is the well-known

$$
\sigma_{\mathrm{opt}}=\pi r^{2}
$$

where $r$ is the radius of the sphere. This equation is valid for this component for all radii exclusive of the region of Rayleigh scattering.

The magnitude of the component of the scattered field due to the creeping wave can be found from the maximum and minimum values of radar cross section of the perfectly conducting sphere. For the radii where these maxima occur, the specular component and creeping wave component are in phase. This yields

where

$$
\sqrt{\sigma_{\mathrm{cw}}}=\sqrt{\sigma_{\mathrm{max}}}-\sqrt{\sigma_{\mathrm{opt}}}
$$

$\sigma_{\mathrm{cw}}=$ component of radar cross section due to the creeping wave, and

$\sigma_{\max }=$ maximum radar cross section for perfectly conducting body.

Similarly

where

$$
\sqrt{\sigma_{\mathrm{ew}}}=\sqrt{\sigma_{\mathrm{opt}}}-\sqrt{\sigma_{\mathrm{min}}}
$$

$\sigma_{\mathrm{m} \text { in }}=$ minimum radar cross section for perfectly conducting body.

Note that $\sigma_{\mathrm{cw}}$ is a function of radius.

The creeping wave field is attenuated by absorption in addition to attenuation by radiation if the conductivity is not infinite. The absorption attenuation factor is taken approximately to be equal to the attenuation factor of a wave traveling along the interface illustrated in figure 2. The attenuation factor for this case is [Ramo and Whinnery, 1947].

where

$$
\alpha=\beta_{0} \frac{\omega \epsilon_{1}}{2 s_{2}}
$$

$\beta_{0}=$ phase factor of ambient medium,

$\epsilon_{1}=$ relative dielectric constant of the ambient medium, and

$s_{2}=$ conductivity of the body.

The attenuation factor is $\alpha=10 \pi, \pi$, and $\pi / 4$ nepers per wavelength for $\epsilon_{r}$ equal to $0-j 0.1,0-j 1.0$ and $0-j 4.0$, respectively. The path of the creeping wave contributing to the monostatic radar cross section is illustrated in figure 1. Its path length is simply $\pi r$. The approximate radar cross section may be written as

and

$$
\sqrt{\sigma_{\max }^{L}}=\sqrt{R^{2} \sigma_{\mathrm{opt}}}+\sqrt{\sigma_{\mathrm{ew}}} e^{-\alpha \pi r}
$$

$$
\sqrt{\sigma_{\min }^{L}}=\sqrt{R^{2} \sigma_{\mathrm{opt}}}-\sqrt{\sigma_{\mathrm{cm}}} e^{-\alpha \pi r}
$$

where

$\sigma_{\max }^{L}$ is the maximum radar cross section of a lossy sphere,

$\sigma_{\min }^{L}$ is the minimum radar cross section of the lossy sphere.

This method has been used to obtain values for the maxima and minima for the three cases. The results are indicated in figure 3 . The attenuation of the creeping wave is such that its contribution is negligible for $\epsilon_{r}=0-j 0.1$ and this is in agreement with the exact solution shown in figure 3 . The agreement between this approximate solution and the exact solution is remarkable for all cases.

It is to be emphasized that the radii associated with these computed values has not been obtained from the approximation. To date no successful method has been obtained for determining the change of the phase of the creeping wave component due to the finite value of the conductivity.

\section{Conclusions}

Two approximate solutions for the radar cross section of the plasma body at the plasma frequency have been presented. In both cases, it is the loss mechanism that controls the radar cross section of such bodies. The simplest approximation does predict the average magnitude but does not properly predict the variations about that average.

These variations may be predicted by the introduction of a loss mechanism in the creeping wave component. An approximate form for the attenuation of the creeping due to loss has been given and its validity demonstrated by means of example.

\section{References}

Franz, W., and K. Depperman (June 1954a), Theorie der Beugung am Kugel unter Beruechsichtigung der Kriechwelle, Ann. Physik 14, 253-264.

Franz, W., and K. Depperman (June 1954b), Theorie der Beugung am Zylinder unter Beruechsichtigung der Krieschwelle, Ann. Physik 14, 361-373.

Keller, J. B., and B. R. Levey (1959), Decay Exponents and Diffraction Coefficients for Surface Waves on Surfaces of Nonconstant Curvature, Special Supplement of Symposium on Electromagnetic Theory, IRE Trans. Ant. Prop. AP- $;$, S52-S61.

Mie, G. (March 1908), Beitraege zur Optik trueber Medien, speciall Kolloider Metalloesunger, Ann. Physik 25, 377-466.

Ramo, S., and J. R. Whinnery (1947), Fields and Waves in Modern Radio, p, 291 (John Wiley \& Sons Inc., New York, N.Y.).

Wait, J. R. (1964), Electromagnetic Surface Waves, Advances in Radio Research 1, 157-217 (Academic Press, London).

(Paper 69D2-458) 